\title{
THE EXTREMAL RANKS AND INERTIAS OF THE LEAST SQUARES SOLUTIONS TO MATRIX EQUATION $A X=B$ SUBJECT TO HERMITIAN CONSTRAINT ${ }^{\dagger}$
}

\author{
LIFANG DAI, MAOLIN LIANG*
}

\begin{abstract}
In this paper, the formulas for calculating the extremal ranks and inertias of the Hermitian least squares solutions to matrix equation $A X=B$ are established. In particular, the necessary and sufficient conditions for the existences of the positive and nonnegative definite solutions to this matrix equation are given. Meanwhile, the least squares problem of the above matrix equation with Hermitian $R$-symmetric and $R$-skew symmetric constraints are also investigated.
\end{abstract}

AMS Mathematics Subject Classification : 15A03, 15A24, 65K15.

Key words and phrases : Matrix equation, rank, inertia, Hermitian solution, least squares solution.

\section{Introduction}

As is well-known, many problems in matrix theory and scientific computing are closely related to the maximal and minimal ranks (abbreviated to extremal ranks) of block matrix or matrix expressions with variant entries. There have been many experts and scholars making their attention to this theme (see, e.g [110]). Recently, Yongge Tian has derived a series of elegant results for calculating the extremal ranks and inertias of some Hermitian linear or nonlinear matrix expressions with one or more variable matrices ([11-15]). In addition, the least square problem of matrix equation has also been studied by using the matrix rank method (see, e.g [16-19]). The following concept was put forward by Trench in $[20]$ :

Let $R \in \mathbb{C}^{n \times n}$ be a nontrivial Hermitian involutory matrix, i.e., $R=R^{*}=$ $R^{-1} \neq I_{n}$. We say that $X \in \mathbb{C}^{n \times n}$ is Hermitian $R$-symmetric ( $R$-skew symmetric) if $X^{*}=X$ and $R X R=X\left(X^{*}=X\right.$ and $\left.R X R=-X\right)$.

Received September 8, 2012. Revised October 5, 2012. Accepted October 7, 2012. * Corresponding author. ${ }^{\dagger}$ This work was supported by the research grants of Education Department of Gansu Province (No.1108B-03) and Tianshui Normal University (No.TSA1104).

(c) 2013 Korean SIGCAM and KSCAM 
Motivated by the work mentioned above, we in this paper investigate the extremal ranks and inertias of the LS solutions of the following matrix equation

$$
A x=B
$$

where $A, B \in \mathbb{C}^{m \times n}$ are given matrices, and the unknown matrix $X \in \mathbb{C}^{n \times n}$ is Hermitian, Hermitian $R$-symmetric, or Hermitian $R$-skew symmetric.

Throughout this paper, $\mathbb{C}_{h}^{n}$ denotes the set of all Hermitian matrices with orders $n$. The symbols $A^{+}, r(A)$ and $\mathcal{R}(A)$ stand for the Moore-Penrose inverse, the rank and the range (column space) of a complex matrix $A$, respectively. Write $A>0(A \geq 0)$ if $A$ is Hermitian positive (nonnegative) definite, and $A>B(A \geq B)$ in the Löwner partial ordering if $A-B$ is positive (nonnegative) definite, where $A$ and $B$ with the same sizes are Hermitian. In addition, the inertia of a Hermitian matrix $M$ is defined to be the triplet

$$
\operatorname{In}(M)=\left\{i_{+}(M), i_{-}(M), i_{0}(M)\right\},
$$

where $i_{+}(M), i_{-}(M)$ and $i_{0}(M)$ are the numbers of the positive, negative and zero eigenvalues of $M$ counted with multiplicities, respectively. Moreover, $E_{A}=$ $I_{m}-A A^{+}$and $F_{A}=I_{n}-A^{+} A$ are two orthogonal projectors of $A \in \mathbb{C}^{m \times n}$.

The remainder of this paper is organized as follows: In section 2 , some results related to rank and inertia will be introduced. In section 3 , the extremal ranks and inertias of the Hermitian LS solutions of matrix equation (1.1) will be derived. Furthermore, the extremal ranks and inertias of the Hermitian $R$ symmetric and $R$-skew symmetric solutions to the LS problem of this matrix equation will be given in section 4 .

\section{Preliminary knowledge}

In this section, we introduce some useful conclusions with respect to the ranks and inertias of block matrices or matrix expressions, which will be utilized in the latter part of this paper.

For the convenience of our expression, we give some results about rank (inertia) of a (Hermitian) matrix as a lemma, which is obvious from the definition.

Lemma 2.1. Let $\mathcal{S}$ be a set consisting of matrices over $\mathbb{C}^{m \times m}$, and $\mathcal{H}$ be a set consisting of Hermitian matrices over $\mathbb{C}_{h}^{m}$. Then

(a) There exists a nonsingular matrix $X \in \mathcal{S}$ if and only if $\max _{X \in \mathcal{S}} r(X)=m$.

(b) All matrices in $\mathcal{S}$ is nonsingular if and only if $\min _{X \in \mathcal{S}} r(X)=m$.

(c) There exists a matrix $X \in \mathcal{H}$ such that $X>0(X<0)$ if and only if $\max _{X \in \mathcal{H}} i_{+}(X)=m\left(\max _{X \in \mathcal{H}} i_{-}(X)=m\right)$.

(d) All $X \in \mathcal{H}$ satisfy $X>0(X<0)$ if and only if $\min _{X \in \mathcal{H}} i_{+}(X)=m$ $\left(\min _{X \in \mathcal{H}} i_{-}(X)=m\right)$.

(e) There exists $X \in \mathcal{H}$ such that $X \geq 0(X \leq 0)$ if and only if $\min _{X \in \mathcal{H}} i_{-}(X)=$ $0\left(\min _{X \in \mathcal{H}} i_{+}(X)=0\right)$.

(f) All $X \in \mathcal{H}$ satisfy $X \geq 0(X \leq 0)$ if and only if $\max _{X \in \mathcal{H}} i_{-}(X)=0$ $\left(\max _{X \in \mathcal{H}} i_{+}(X)=0\right)$. 
Lemma $2.2([5])$. Let $A \in \mathbb{C}^{m \times n}, B \in \mathbb{C}^{m \times k}$ and $C \in \mathbb{C}^{l \times n}$. Then

$$
\begin{aligned}
& r\left[\begin{array}{ll}
A & B
\end{array}\right]=r(A)+r\left(E_{A} B\right)=r(B)+r\left(E_{B} A\right), \\
& r\left[\begin{array}{l}
A \\
C
\end{array}\right]=r(A)+r\left(C F_{A}\right)=r(C)+r\left(A F_{C}\right), \\
& r\left[\begin{array}{ll}
A & B \\
C & O
\end{array}\right]=r(B)+r(C)+r\left(E_{B} A F_{C}\right) .
\end{aligned}
$$

Lemma $2.3([12])$. Let $M=\left[\begin{array}{cc}A & B \\ B^{*} & 0\end{array}\right]$, where $A \in \mathbb{C}_{h}^{m}, B \in \mathbb{C}^{m \times n}$ are given.

Then $i_{ \pm}(M)=r(B)+i_{ \pm}\left(E_{B} A E_{B}\right)$.

Lemma 2.4 ([11, 12]). Suppose that the conditions are the same as in Lemma 2.3. Then

$$
\begin{aligned}
\max _{X \in \mathbb{C}_{h}^{n}} r\left(A-B X B^{*}\right) & =r[A B], \quad \min _{X \in \mathbb{C}_{h}^{n}} r\left(A-B X B^{*}\right)=2 r[A B]-r(M), \\
\max _{X \in \mathbb{C}_{h}^{n}} i_{ \pm}\left(A-B X B^{*}\right) & =i_{ \pm}(M), \quad \min _{X \in \mathbb{C}_{h}^{n}} i_{ \pm}\left(A-B X B^{*}\right)=r[A B]-i_{\mp}(M) .
\end{aligned}
$$

Lemma 2.5 ([12]). Let $A, B, C, D$ and $P, Q$ be matrices such that the expressions $D-C A^{+} B$ and $D-C P^{+} A Q^{+} B$ are defined, respectively. Then

$$
\begin{gathered}
r\left(D-C A^{+} B\right)=r\left[\begin{array}{cc}
A^{*} A A^{*} & A^{*} B \\
C A^{*} & D
\end{array}\right]-r(A) . \\
r\left(D-C P^{+} A Q^{+} B\right)=r\left[\begin{array}{ccc}
P^{*} A Q^{*} & P^{*} P P^{*} & 0 \\
Q^{*} Q Q^{*} & 0 & Q^{*} B \\
0 & C P^{*} & -D
\end{array}\right]-r(P)-r(Q) .
\end{gathered}
$$

Particularly, if $A \in \mathbb{C}_{h}^{m}, B \in \mathbb{C}^{m \times n}$ and $D \in \mathbb{C}_{h}^{m}$. Then

$$
i_{ \pm}\left(D-B^{*} A^{+} B\right)=i_{ \pm}\left[\begin{array}{cc}
A^{3} & A B \\
(A B)^{*} & D
\end{array}\right]-i_{ \pm}(A) .
$$

Lemma 2.6 ([12]). Let $A \in \mathbb{C}_{h}^{m}, B \in \mathbb{C}_{h}^{n}$ and $P, Q \in \mathbb{C}^{m \times n}$. Then $i_{ \pm}\left(P^{*} A P\right) \leq$ $i_{ \pm}(A)$. In particular,

(a) $r\left(P^{*} A P\right)=r(A)$ if and only if $i_{+}\left(P^{*} A P\right)=i_{+}(A), i_{-}\left(P^{*} A P\right)=i_{-}(A)$.

(b) If $P^{*} A P=B$ and $Q^{*} B Q=A$, then $i_{ \pm}(A)=i_{ \pm}(B)$ and $r(A)=r(B)$.

Lemma $2.7([21])$. Let $A \in^{m \times n}, B \in \mathbb{C}^{m \times k}$ and $C \in \mathbb{C}^{l \times k}$ be given. Then,

$$
\begin{aligned}
& \max _{X \in \mathbb{C}^{k \times l}} r(A-B X C)=\min \left\{r[A B], r\left[\begin{array}{l}
A \\
C
\end{array}\right]\right\}, \\
& \min _{X \in \mathbb{C}^{k \times l}} r(A-B X C)=r[A B]+r\left[\begin{array}{l}
A \\
C
\end{array}\right]-r\left[\begin{array}{ll}
A & B \\
C & 0
\end{array}\right] .
\end{aligned}
$$




\section{Extremal ranks and inertias of the Hermitian LS solutions to matrix equation (1.1)}

The following lemma quoted from Trench [20] is necessary.

Lemma 3.1. Let $A, B \in \mathbb{C}^{m \times n}$ be given matrices, and assume that rank $(A)=k$ and $A=\Phi \Sigma \Omega^{*}$ is the singular value decomposition of $A$, where $\Phi \in \mathbb{C}^{m \times m}$ and $\Omega \in \mathbb{C}^{n \times n}$ are unitary matrices, $\Sigma=\operatorname{diag}\left(\sigma_{1}, \sigma_{2}, \ldots, \sigma_{k}\right)>0$ and $\sigma_{j}$ is the singular value. Define

$$
S=\left(s_{i j}\right)=\Phi^{*}\left(B A^{*}-A B^{*}\right) \Phi,
$$

then the Hermitian LS solutions to matrix equation (1.1) can be expressed as

$$
\begin{gathered}
X=X_{0}-\frac{1}{2} \Omega T \Omega^{*}+F_{A} H F_{A}, \\
\text { where } X_{0}=\left(I_{n}-\frac{1}{2} A^{+} A\right)\left(A^{+} B\right)^{*}+A^{+} B\left(I_{n}-\frac{1}{2} A^{+} A\right), T=\left(t_{i j}\right) \in \mathbb{C}_{h}^{n}, \\
t_{i j}= \begin{cases}\frac{\sigma_{j}^{2}-\sigma_{i}^{2}}{\sigma_{i} \sigma_{j}\left(\sigma_{i}^{2}+\sigma_{j}^{2}\right)} s_{i j} & 1 \leq i, j \leq k, \\
0, & \text { otherwise, }\end{cases}
\end{gathered}
$$

and $H \in \mathbb{C}_{h}^{n}$ is arbitrary. Particularly, if $A B^{*}=B A^{*}$, then $T=0$ in (3.1).

Let $\mathcal{S}_{1}$ be the collections of the Hermitian LS solutions of matrix equation (1.1), namely,

$$
\mathcal{S}_{1}=\left\{X \mid\|A X-B\|=\min , X \in \mathbb{C}_{h}^{n}\right\}
$$

.Hence, we have the following theorem.

Theorem 3.1. Let $A, B$ be given matrices as in (1.1). Setting

$$
\begin{aligned}
T_{1} & =\left[\begin{array}{cccccc}
A B^{*} A & 0 & 0 & A A^{*} A & 0 & 0 \\
0 & A^{*} B A^{*} & 0 & 0 & A^{*} A A^{*} & 0 \\
A A^{*} A & 0 & 0 & 0 & 0 & A \\
0 & A^{*} A A^{*} & 0 & 0 & 0 & A^{*} A \\
0 & 0 & A^{*} A A^{*} & 0 & 0 & A^{*} B \\
0 & 0 & 2 A^{*} & -A^{*} A & A^{*} & \Omega T \Omega^{*}
\end{array}\right], \\
T_{2} & =\left[\begin{array}{ccc}
0 & A^{*} A A^{*} & A^{*} \\
A A^{*} A & 0 & A A^{*} B A^{*} \\
A & A B^{*} A A^{*} & A \Omega T \Omega^{*} A^{*}
\end{array}\right] .
\end{aligned}
$$

Then

$$
\begin{gathered}
\max _{X \in \mathcal{S}_{1}} r(X)=n+r\left(T_{1}\right)-6 r(A), \\
\min _{X \in \mathcal{S}_{1}} r(X)=2 r\left(T_{1}\right)-r\left(T_{2}\right)-8 r(A),
\end{gathered}
$$




$$
\begin{gathered}
\max _{X \in \mathcal{S}_{1}} i_{ \pm}(X)=n+i_{\mp}\left(T_{2}\right)-2 r(A), \\
\min _{X \in \mathcal{S}_{1}} i_{ \pm}(X)=r\left(T_{1}\right)-i_{ \pm}\left(T_{2}\right)-4 r(A) .
\end{gathered}
$$

In consequence,

(a) There exists a nonsingular matrix $X \in \mathcal{S}_{1}$ if and only if $r\left(T_{1}\right)=6 r(A)$.

(b) All matrices in $\mathcal{S}_{1}$ is nonsingular if and only if $2 r\left(T_{1}\right)=n+r\left(T_{2}\right)+8 r(A)$.

(c) There exists a matrix $X \in \mathcal{S}_{1}$ such that $X>0(X<0)$ if and only if $i_{-}\left(T_{2}\right)=2 r(A)\left(i_{+}\left(T_{2}\right)=2 r(A)\right)$.

(d) Any matrix $X \in \mathcal{S}_{1}$ satisfy $X>0(X<0)$ if and only if $r\left(T_{1}\right)=n+$ $i_{+}\left(T_{2}\right)+4 r(A)\left(r\left(T_{1}\right)=n+i_{-}\left(T_{2}\right)+4 r(A)\right)$.

(e) There exists a matrix $X \in \mathcal{S}_{1}$ such that $X \geq 0$ ( $\left.X \leq 0\right)$ if and only if $r\left(T_{1}\right)=i_{-}\left(T_{2}\right)+4 r(A)\left(r\left(T_{1}\right)=i_{+}\left(T_{2}\right)+4 r(A)\right)$.

(f) Any matrix $X \in \mathcal{S}_{1}$ satisfy $X \geq 0(X \leq 0)$ if and only if $n+i_{+}\left(T_{2}\right)=2 r(A)$ $\left(n+i_{-}\left(T_{2}\right)=2 r(A)\right)$.

Proof. Applying Lemma 2.4 to $X$ as given in (3.1), we obtain that

$$
\begin{aligned}
& \max _{H \in \mathbb{C}_{h}^{n}} r(X)=r\left[-N, F_{A}\right], \quad \min _{H \in \mathbb{C}_{h}^{n}} r(X)=2 r\left[-N, F_{A}\right]-r(M) . \\
& \max _{H \in \mathbb{C}_{h}^{n}} i_{ \pm}(X)=i_{\mp}(M), \quad \min _{H \in \mathbb{C}_{h}^{n}} i_{ \pm}(X)=r\left[-N, F_{A}\right]-i_{ \pm}(M) .
\end{aligned}
$$

where

$$
M=\left(\begin{array}{cc}
-N & F_{A} \\
F_{A} & 0
\end{array}\right)
$$

and

$$
N=X_{0}-\frac{1}{2} \Omega T \Omega^{*}
$$

Now we simplify $r\left[N, F_{A}\right]$ and $i_{ \pm}(M)$ by the three types of elementary block matrix operations, and elementary block congruence matrix operations (see [12] for details), respectively.

It follows from Lemma 2.2 that

$$
\begin{aligned}
r\left[N, F_{A}\right]= & n-r(A) \\
& +r\left[A^{+} B+\frac{1}{2}\left(A^{+} A B^{*}\left(A^{+}\right)^{*}-A^{+} B A^{+} A-A^{+} A \Omega T \Omega^{*}\right)\right] .
\end{aligned}
$$


Note that $A^{+} A \Omega T \Omega^{*}=\Omega T \Omega^{*}$, then applying the formula (2.2) in Lemma 2.5 to the third item in (3.7), we obtain

$$
\begin{aligned}
& r\left[A^{+} B+\frac{1}{2}\left(A^{+} A B^{*}\left(A^{+}\right)^{*}-A^{+} B A^{+} A-A^{+} A \Omega T \Omega^{*}\right)\right] \\
& =r\left[-\Omega T \Omega^{*}+2 A^{+} B+A^{*}\left(A^{*}\right)^{+} B^{*}\left(A^{+}\right)^{*}-A^{+} B A^{+} A\right] \\
& =r\left[\begin{array}{ccccccc}
A^{*} B & 0 & 0 & A^{*} A A^{*} & 0 & 0 & 0 \\
0 & A B^{*} A & 0 & 0 & A A^{*} A & 0 & 0 \\
0 & 0 & A^{*} B A^{*} & 0 & 0 & A^{*} A A^{*} & 0 \\
I_{n} & 0 & 0 & 0 & 0 & 0 & -I_{n} \\
0 & A A^{*} A & 0 & 0 & 0 & 0 & A \\
0 & 0 & A^{*} A A^{*} & 0 & 0 & 0 & A^{*} A \\
0 & 0 & 0 & 2 A^{*} & -A^{*} A & A^{*} & \Omega T \Omega^{*}
\end{array}\right]-5 r(A) \\
& =r\left(T_{1}\right)-5 r(A) .
\end{aligned}
$$

Moreover, applying Lemma 2.3 to the block matrix $M$ and simplifying by Lemma 2.6 (b) follow that

$$
\begin{aligned}
& i_{ \pm}(M)=r\left(F_{A}\right)+i_{\mp}\left(A^{+} A N A^{+} A\right) \\
& =i_{\mp}\left[A^{+} A B^{*}\left(A^{+}\right)^{*}+A^{+} B A^{*}\left(A^{+}\right)^{*}-A^{+} A \Omega T \Omega^{*} A^{*}\left(A^{+}\right)^{*}\right]+n-r(A) \\
& =i_{\mp}\left[A B^{*} A A^{+}+A A^{+} B A^{*}-A \Omega T \Omega^{*} A^{*}\right]+n-r(A) \\
& =i_{ \pm}\left[A \Omega T \Omega^{*} A^{*}-A B^{*} A A^{+}-A A^{+} B A^{*}\right]+n-r(A) .
\end{aligned}
$$

From (2.3), we derive

$$
\begin{aligned}
& i_{ \pm}\left[A \Omega T \Omega^{*} A^{*}-A B^{*} A A^{+}-A A^{+} B A^{*}\right] \\
& =i_{ \pm}\left[A \Omega T \Omega^{*} A^{*}-\left[A B^{*} A I_{m}\right]\left[\begin{array}{ll}
A & A^{*}
\end{array}\right]^{+}\left[\begin{array}{c}
A^{*} B A^{*} \\
I_{m}
\end{array}\right]\right] \\
& =i_{ \pm}\left[\begin{array}{cc}
A & A^{*} \\
A &
\end{array}\right]^{3}\left[\begin{array}{cc}
A & A^{*}
\end{array}\right]\left[\begin{array}{c}
A^{*} B A^{*} \\
I_{m}
\end{array}\right] \\
& {\left[A B^{*} A I_{m}\right]\left[\begin{array}{ll}
A & A^{*}
\end{array}\right]-i \pm\left[\begin{array}{ll}
A & A^{*}
\end{array}\right]} \\
& =i_{ \pm}\left(T_{2}\right)-r(A) .
\end{aligned}
$$

In addition, since $M$ and $T_{2}$ are Hermitian, then

$$
r(M)=i_{+}(M)+i_{-}(M) \operatorname{and} r\left(T_{2}\right)=i_{+}\left(T_{2}\right)+i_{-}\left(T_{2}\right) .
$$

Substituting (3.8)-(3.12) into (3.6)-(3.7) deduces that (3.2)-(3.5) hold. And the results (a)-(f) follow from applying Lemma 2.1 to (3.2)-(3.5).

If the matrices $A$ and $B$ given in Theorem 3.1 satisfy $A B^{*}=B A^{*}$, then Theorem 3.1 can be simplified, that is, 
Corollary 3.2. Let $A B^{*}=B A^{*}$ in Theorem 3.1, then

$$
\begin{gathered}
\max _{X \in \mathcal{S}} r(X)=n-r(A)+r\left(A^{*} B\right), \\
\min _{X \in \mathcal{S}} r(X)=2 r\left(A^{*} B\right)-r\left(A B^{*}\right), \\
\max _{X \in \mathcal{S}} i_{ \pm}(X)=n-r(A)+i_{ \pm}\left(A B^{*}\right), \\
\min _{X \in \mathcal{S}} i \pm(X)=r\left(A^{*} B\right)-i_{\mp}\left(A B^{*}\right) .
\end{gathered}
$$

In consequence,

(a) There exists a nonsingular matrix $X \in \mathcal{S}$ if and only if $r(A)=r\left(A^{*} B\right)$.

(b) All matrices in $\mathcal{S}$ is nonsingular if and only if $2 r\left(A^{*} B\right)=n+r\left(A B^{*}\right)$.

(c) There exists a matrix $X \in \mathcal{S}$ such that $X>0(X<0)$ if and only if $i_{+}\left(A B^{*}\right)=r(A)\left(i_{-}\left(A B^{*}\right)=r(A)\right)$.

(d) Any matrix $X \in \mathcal{S}$ satisfy $X>0(X<0)$ if and only if $r\left(A^{*} B\right)=n+$ $i_{-}\left(A B^{*}\right)\left(r\left(A^{*} B\right)=n+i_{+}\left(A B^{*}\right)\right)$.

(e) There exists a matrix $X \in \mathcal{S}$ such that $X \geq 0$ ( $X \leq 0)$ if and only if $r\left(A^{*} B\right)=n+i_{+}\left(A B^{*}\right)\left(r\left(A^{*} B\right)=n+i_{-}\left(A B^{*}\right)\right)$.

(f) Any matrix $X \in \mathcal{S}$ satisfy $X \geq 0(X \leq 0)$ if and only if $r(A)=n+i_{-}\left(A B^{*}\right)$ $\left(r(A)=n+i_{+}\left(A B^{*}\right)\right)$.

Proof. From Lemma 3.1, $A B^{*}=B A^{*}$ deduces $T=0$ in (3.1). Then we get

$$
\begin{aligned}
& r\left[N, F_{A}\right]=n-r(A)+r\left[A^{+} B+\frac{1}{2}\left(A^{+} A B^{*}\left(A^{+}\right)^{*}-A^{+} B A^{+} A-A^{+} A \Omega T \Omega^{*}\right)\right] \\
& =n-r(A)+r\left(A^{+} B\right)=n-r(A)+r\left(A^{*} B\right), \\
& i_{ \pm}(M)=r\left(F_{A}\right)+i_{\mp}\left(A^{+} A N A^{+} A\right)=n-r(A)+i_{\mp}\left[A^{+} A B^{*}\left(A^{+}\right)^{*}\right] \\
& =n-r(A)+i_{\mp}\left(A B^{*} A A^{+}\right) \\
& =n-r(A)+i_{\mp}\left(A B^{*}\right),
\end{aligned}
$$

and

$$
r(M)=2 n-2 r(A)+r\left(A B^{*}\right) .
$$

Therefore, substituting (3.17)-(3.19) into (3.6)-(3.7) yields (3.13)-(3.16).

In addition, if $A B^{*}=B A^{*}$ and $A A^{+} B=B$, or equivalently, $A B^{*}=B A^{*}$ and $\mathcal{R}(B) \subseteq \mathcal{R}(A)$, i.e., matrix equation (1.1) is consistent for Hermitian $X$. In this case, it is easy to verify that $r\left(A^{*} B\right)=r(B)$, substituting it into the formulas (3.13)-(3.16), then we obtain the same results as proposed in Tian [12]. 
Corollary 2. Assume that matrix equation (1.1) is consistent for Hermitian $X$, that is, $A B^{*}=B A^{*}$ and $A A^{+} B=B$, then,

$$
\begin{aligned}
\max _{A X=B, X \in \mathbb{C}_{h}^{n}} r(X) & =n-r(A)+r(B)\}, \quad \min _{A X=B, X \in \mathbb{C}_{h}^{n}} r(X)=2 r(B)-r\left(A B^{*}\right) . \\
\max _{A X=B, X \in \mathbb{C}_{h}^{n}} i_{ \pm}(X) & =n-r(A)+i_{ \pm}\left(A B^{*}\right), \min _{A X=B, X \in \mathbb{C}_{h}^{n}} i_{ \pm}(X)=r(B)-i_{\mp}\left(A B^{*}\right) .
\end{aligned}
$$

Remark 3.1. The extremal ranks and inertias of the Hermitian LS solutions to matrix equations $A X=B, X C=D$ can be obtained by Theorem 3.1. In fact, let $\widetilde{A}=\left[\begin{array}{c}A \\ C^{*}\end{array}\right]$ and $\widetilde{B}=\left[\begin{array}{c}B \\ D^{*}\end{array}\right]$. Then the LS problem of matrix equations $A X=B, X C=D$ is equivalent to that of matrix equation $\widetilde{A} X=\widetilde{B}, X \in \mathbb{C}_{h}^{n}$.

\section{Extremal ranks and inertias for Hermitian $R$-symmetric and $R$-skew symmetric LS problems}

In this section, as application of Theorem 3.1, we study the extremal ranks and inertias of the Hermitian $R$-symmetric and Hermitian $R$-skew symmetric LS solutions of matrix equation (1.1), respectively.

Let $R \in \mathbb{C}^{n \times n}$ be a nontrivial Hermitian involution. Assume that positive integers $s$ and $t$ are respectively the dimensions of the eigenspaces of $R$ associated with the eigenvalues $\lambda=1$ and $\lambda=-1$, thus $s+t=n$. Then, from [20], we know that

$X \in \mathbb{C}^{n \times n}$ is Hermitian $R$-symmetric if and only if

$$
X=\left[\begin{array}{ll}
P & Q
\end{array}\right]\left[\begin{array}{ll}
X_{1} & \\
& X_{2}
\end{array}\right]\left[\begin{array}{l}
P^{*} \\
Q^{*}
\end{array}\right],
$$

and $X \in \mathbb{C}^{n \times n}$ is Hermitian $R$-skew symmetric if and only if

$$
X=\left[\begin{array}{ll}
P & Q
\end{array}\right]\left[\begin{array}{ll} 
& X_{3} \\
X_{3}^{*} &
\end{array}\right]\left[\begin{array}{l}
P^{*} \\
Q^{*}
\end{array}\right],
$$

where $P \in \mathbb{C}^{n \times s}$ consists of the ortho-normal bases of the eigen-space to $\lambda=1$, and $Q \in \mathbb{C}^{n \times t}$ to $\lambda=-1 . X_{1} \in \mathbb{C}_{h}^{s}, X_{2} \in \mathbb{C}_{h}^{t}, X_{3} \in \mathbb{C}^{s \times t}$ are arbitrary.

For matrices $A, B \in \mathbb{C}^{m \times n}$ as given in (1.1), denote

$$
[P Q]=\left[\begin{array}{ll}
A_{1} & A_{2}
\end{array}\right] \text { and } B[P Q]=\left[\begin{array}{ll}
B_{1} & B_{2}
\end{array}\right]
$$

with $A_{1}, B_{1} \in \mathbb{C}^{m \times s}$ and $A_{2}, B_{2} \in \mathbb{C}^{m \times t}$. Combining the equalities (4.1)-(4.3) with (1.1), we know that the Hermitian $R$-symmetric LS problem of (1.1) is equivalent to that of the following matrix equations

$$
A_{1} X_{1}=B_{1} \text { and } A_{2} X_{2}=B_{2} \text { with } X_{1} \in \mathbb{C}_{h}^{s}, X_{2} \in \mathbb{C}_{h}^{t} .
$$

And the Hermitian $R$-skew symmetric LS problem of (1.1) is equivalent to that of the following matrix equations

$$
A_{1} X_{3}=B_{2} \text { and } A_{2} X_{3}^{*}=B_{1} \text { with } X_{3} \in \mathbb{C}^{s \times t} .
$$


We need the following lemmas.

Lemma 4.1 ([20]). Let $A, B \in \mathbb{C}^{m \times n}$ be given matrices having the partition as in (4.3), and $R \in \mathbb{C}^{n \times n}$ be a nontrx ivial unitary involution. For $j=1,2$, assume that $A_{j}=\Phi_{j} \Sigma_{j} \Omega_{j}^{*}$ is the singular value decomposition of $A_{j}$, where $\Phi_{j} \in \mathbb{C}^{m \times m}, \Omega_{1} \in \mathbb{C}^{s \times s}$ and $\Omega_{2} \in \mathbb{C}^{t \times t}$ are unitary matrices, $\Sigma_{j}$ consisting of the singular values of $A_{j}$ is a diagonal matrix. Define

$$
S_{j}=\Phi_{j}^{*}\left(B_{j} A_{j}^{*}-A_{j} B_{j}^{*}\right) \Phi_{j} .
$$

Then the Hermitian R-symmetric LS solutions of matrix equation (1.1) can be written as in (4.1), and

$$
X_{j}=\bar{X}_{j}-\frac{1}{2} \Omega_{j} T_{j} \Omega_{j}^{*}+F_{A_{j}} H_{j} F_{A_{j}}, j=1,2,
$$

where $\bar{X}_{1}=\left(I_{s}-\frac{1}{2} A_{1}^{+} A_{1}\right)\left(A_{1}^{+} B_{1}\right)^{*}+A_{1}^{+} B_{1}\left(I_{s}-\frac{1}{2} A_{1}^{+} A_{1}\right)$,

$\bar{X}_{2}=\left(I_{t}-\frac{1}{2} A_{2}^{+} A_{2}\right)\left(A_{2}^{+} B_{2}\right)^{*}+A_{2}^{+} B_{2}\left(I_{t}-\frac{1}{2} A_{2}^{+} A_{2}\right), T_{j}$ related to $S_{j}$ and $\Sigma_{j}(j=$ $1,2)$ is a fixed matrix. $H_{1} \in \mathbb{C}_{h}^{s}$ and $H_{2} \in \mathbb{C}_{h}^{t}$ are arbitrary.

Particularly, if $A_{j} B_{j}^{*}=B_{j} A_{j}^{*}$, then $T_{j}=0$.

Lemma 4.2 ([20]). Suppose that the conditions are the same as in Lemma 4.1. Define $\quad S_{3}=\Phi_{1}^{*}\left(B_{2} A_{2}^{*}-A_{1} B_{1}^{*}\right) \Phi_{2}$,

then the Hermitian $R$-skew symmetric $L S$ solutions of matrix equation (1.1) can be expressed as in (4.2), and

$$
X_{3}=\bar{X}_{3}-\Omega_{1} T_{3} \Omega_{2}^{*}+F_{A_{1}} H_{3} F_{A_{2}},
$$

where $\bar{X}_{3}=A_{1}^{+} B_{2}+F_{A_{1}}\left(A_{2}^{+} B_{1}\right)^{*}, T_{3}$ related to $S_{3}$ and $\Sigma_{j}(j=1,2)$ is a fixed matrix, and $H_{3} \in \mathbb{C}^{s \times t}$ is arbitrary. Particularly, if $B_{2} A_{2}^{*}=A_{1} B_{1}^{*}$, then $T_{3}=0$.

$\mathcal{S}_{2}=\left\{X \mid\|A X-B\|=\min , X \in \mathbb{C}_{h}^{n}\right.$ and $\left.R X R=X\right\}$.

Then from Lemma 4.1, we have the following theorem.

Theorem 4.1. Given matrices $A, B \in \mathbb{C}^{m \times n}$, and the nontrivial involution $R \in \mathbb{C}^{n \times n}$. For $j=1,2, \Omega_{j}, T_{j}$ as in Lemma 4.1, let

$$
\begin{aligned}
T_{j 1} & =\left[\begin{array}{cccccc}
A_{j} B_{j}^{*} A_{j} & 0 & 0 & A_{j} A_{j}^{*} A_{j} & 0 & 0 \\
0 & A_{j}^{*} B_{j} A_{j}^{*} & 0 & 0 & A_{j}^{*} A_{j} A_{j}^{*} & 0 \\
A_{j} A_{j}^{*} A_{j} & 0 & 0 & 0 & 0 & A_{j} \\
0 & A_{j}^{*} A_{j} A_{j}^{*} & 0 & 0 & 0 & A_{j}^{*} A_{j} \\
0 & 0 & A_{j}^{*} A_{j} A_{j}^{*} & 0 & 0 & A_{j}^{*} B_{j} \\
0 & 0 & 2 A_{j}^{*} & -A_{j}^{*} A_{j} & A_{j}^{*} & \Omega_{j} T_{j} \Omega_{j}^{*}
\end{array}\right], \\
T_{j 2}= & {\left[\begin{array}{ccc}
0 & A_{j}^{*} A_{j} A_{j}^{*} & A_{j}^{*} \\
A_{j} A_{j}^{*} A_{j} & 0 & A_{j} A_{j}^{*} B_{j} A_{j}^{*} \\
A_{j} & A_{j} B_{j}^{*} A_{j} A_{j}^{*} & A_{j} \Omega_{j} T_{j} \Omega_{j}^{*} A_{j}^{*}
\end{array}\right], }
\end{aligned}
$$

then, 


$$
\begin{aligned}
\max _{X \in \mathcal{S}_{2}} r(X) & =s+t+r\left(T_{11}\right)+r\left(T_{12}\right)-6 r\left(A_{1}\right)-6 r\left(A_{2}\right), \\
\min _{X \in \mathcal{S}_{2}} r(X) & =2 r\left(T_{11}\right)+2 r\left(T_{21}\right)-r\left(T_{12}\right)-r\left(T_{22}\right)-8 r\left(A_{1}\right)-8 r\left(A_{2}\right), \\
\max _{X \in \mathcal{S}_{2}} i_{ \pm}(X) & =s+t+i_{\mp}\left(T_{12}\right)+i_{\mp}\left(T_{22}\right)-2 r\left(A_{1}\right)-2 r\left(A_{2}\right), \\
\min _{X \in \mathcal{S}_{2}} i_{ \pm}(X) & =r\left(T_{11}\right)+r\left(T_{21}\right)-i_{ \pm}\left(T_{12}\right)-i_{ \pm}\left(T_{22}\right)-4 r\left(A_{1}\right)-4 r\left(A_{2}\right) .
\end{aligned}
$$

In consequence,

(a) There exists a nonsingular matrix $X \in \mathcal{S}_{2}$ if and only if $r\left(T_{j 1}\right)=6 r\left(A_{j}\right)$ for $j=1,2$.

(b) All matrices in $\mathcal{S}_{2}$ is nonsingular if and only if $2 r\left(T_{11}\right)=s+r\left(T_{12}\right)+8 r\left(A_{1}\right)$ and $2 r\left(T_{21}\right)=t+r\left(T_{22}\right)+8 r\left(A_{2}\right)$.

(c) There exists a matrix $X \in \mathcal{S}_{2}$ such that $X>0(X<0)$ if and only if $i_{-}\left(T_{12}\right)=2 r\left(A_{1}\right)$ and $i_{-}\left(T_{22}\right)=2 r\left(A_{2}\right)\left(i_{+}\left(T_{12}\right)=2 r\left(A_{1}\right)\right.$ and $i_{+}\left(T_{22}\right)=$ $\left.2 r\left(A_{2}\right)\right)$.

(d) Any matrix $X \in \mathcal{S}_{2}$ satisfy $X>0(X<0)$ if and only if $r\left(T_{11}\right)=s+$ $i_{+}\left(T_{12}\right)+4 r\left(A_{1}\right)$ and $r\left(T_{21}\right)=t+i_{+}\left(T_{22}\right)+4 r\left(A_{2}\right)\left(r\left(T_{11}\right)=t+i_{-}\left(T_{12}\right)+4 r\left(A_{1}\right)\right.$ and $\left.r\left(T_{21}\right)=t+i_{-}\left(T_{22}\right)+4 r\left(A_{2}\right)\right)$.

(e) There exists a matrix $X \in \mathcal{S}_{2}$ such that $X \geq 0(X \leq 0)$ if and only if $r\left(T_{11}\right)=$ $i_{-}\left(T_{12}\right)+4 r\left(A_{1}\right)$ or $r\left(T_{21}\right)=i_{-}\left(T_{22}\right)+4 r\left(A_{2}\right)\left(r\left(T_{11}\right)=i_{+}\left(T_{12}\right)+4 r\left(A_{1}\right)\right.$ or $\left.r\left(T_{21}\right)=i_{+}\left(T_{22}\right)+4 r\left(A_{2}\right)\right)$.

(f) Any matrix $X \in \mathcal{S}_{2}$ satisfy $X \geq 0(X \leq 0)$ if and only if $s+i_{+}\left(T_{12}\right)=2 r\left(A_{1}\right)$ or $t+i_{+}\left(T_{22}\right)=2 r\left(A_{2}\right)\left(s+i_{-}\left(T_{12}\right)=2 r\left(A_{1}\right)\right.$ or $\left.t+i_{-}\left(T_{22}\right)=2 r\left(A_{2}\right)\right)$.

Proof. . It follows from Lemma 4.1 and (4.1) that

$$
r(X)=r\left(X_{1}\right)+r\left(X_{2}\right) \text { and } i_{ \pm}(X)=i_{ \pm}\left(X_{1}\right)+i_{ \pm}\left(X_{2}\right) .
$$

Applying Theorem 3.1 to $X_{j}$ as given in (4.6) gives the extremal ranks and inertias of Hermitian LS solutions to matrix equation (4.4), then (4.12) implies that (4.8)-(4.11) hold. And the conclusions (a)-(f) are obvious by applying Lemma 2.1 to (4.8)-(4.11).

It is noteworthy that the results expressed in Theorem 4.1 will be simplified when adding the hypotheses $A_{j} B_{j}^{*}=B_{j} A_{j}^{*}$ and $A_{j} A_{j}^{+} B_{j}=B_{j}$ for $j=1,2$, the proofs of which are omitted.

Corollary 3. Suppose that $A_{j} B_{j}^{*}=B_{j} A_{j}^{*}, j=1,2$ in Theorem 4.1, then,

$$
\begin{gathered}
\max _{X \in \mathcal{S}_{2}} r(X)=s+t-r\left(A_{1}\right)-r\left(A_{2}\right)+r\left(A_{1}^{*} B_{1}\right)+r\left(A_{2}^{*} B_{2}\right) . \\
\min _{X \in \mathcal{S}_{2}} r(X)=2 r\left(A_{1}^{*} B_{1}\right)+2 r\left(A_{2}^{*} B_{2}\right)-r\left(A_{1} B_{1}^{*}\right)-r\left(A_{2} B_{2}^{*}\right) . \\
\max _{X \in \mathcal{S}_{2}} i_{ \pm}(X)=s+t-r\left(A_{1}\right)-r\left(A_{2}\right)+i_{ \pm}\left(A_{1} B_{1}^{*}\right)+i_{ \pm}\left(A_{2} B_{2}^{*}\right) .
\end{gathered}
$$




$$
\min _{X \in \mathcal{S}_{2}} i \pm(X)=r\left(A_{1}^{*} B_{1}\right)+r\left(A_{2}^{*} B_{2}\right)-i_{\mp}\left(A_{1} B_{1}^{*}\right)-i_{\mp}\left(A_{2} B_{2}^{*}\right) .
$$

Moreover,

(a) There exists a nonsingular matrix $X \in \mathcal{S}_{2}$ if and only if $r\left(A_{1}\right)=r\left(A_{1}^{*} B_{1}\right)$ and $r\left(A_{2}\right)=r\left(A_{2}^{*} B_{2}\right)$.

(b) All matrices in $\mathcal{S}_{2}$ is nonsingular if and only if $2 r\left(A_{1}^{*} B_{1}\right)=s+r\left(A_{1} B_{1}^{*}\right)$ and $2 r\left(A_{2}^{*} B_{2}\right)=t+r\left(A_{2} B_{2}^{*}\right)$.

(c) There exists a matrix $X \in \mathcal{S}_{2}$ such that $X>0(X<0)$ if and only if $i_{+}\left(A_{1} B_{1}^{*}\right)=r\left(A_{1}\right)$ and $i_{+}\left(A_{2} B_{2}^{*}\right)=r\left(A_{2}\right)\left(i_{-}\left(A_{1} B_{1}^{*}\right)=r\left(A_{1}\right)\right.$ and $i_{-}\left(A_{2} B_{2}^{*}\right)=$ $\left.r\left(A_{2}\right)\right)$.

(d) Any matrix $X \in \mathcal{S}_{2}$ satisfy $X>0(X<0)$ if and only if $r\left(A_{1}^{*} B_{1}\right)=$ $s+i_{-}\left(A_{1} B_{1}^{*}\right)$ and $r\left(A_{2}^{*} B_{2}\right)=t+i_{-}\left(A_{2} B_{2}^{*}\right)\left(r\left(A_{1}^{*} B_{1}\right)=s+i_{+}\left(A_{1} B_{1}^{*}\right)\right.$ and $\left.r\left(A_{2}^{*} B_{2}\right)=t+i_{+}\left(A_{2} B_{2}^{*}\right)\right)$.

(e) There exists a matrix $X \in \mathcal{S}_{2}$ such that $X \geq 0$ ( $\left.X \leq 0\right)$ if and only if $r\left(A_{1}^{*} B_{1}\right)=s+i_{+}\left(A_{1} B_{1}^{*}\right)$ or $r\left(A_{2}^{*} B_{2}\right)=t+i_{+}\left(A_{2} B_{2}^{*}\right)\left(r\left(A_{1}^{*} B_{1}\right)=s+i_{-}\left(A_{1} B_{1}^{*}\right)\right.$ or $\left.r\left(A_{2}^{*} B_{2}\right)=t+i_{-}\left(A_{2} B_{2}^{*}\right)\right)$.

(f) Any matrix $X \in \mathcal{S}_{2}$ satisfy $X \geq 0(X \leq 0)$ if and only if $r\left(A_{1}\right)=s+$ $i_{-}\left(A_{1} B_{1}^{*}\right)$ or $r\left(A_{2}\right)=t+i_{-}\left(A_{2} B_{2}^{*}\right)\left(r\left(A_{1}\right)=s+i_{+}\left(A_{1} B_{1}^{*}\right)\right.$ or $r\left(A_{2}\right)=t+$ $\left.i_{+}\left(A_{2} B_{2}^{*}\right)\right)$.

Corollary 4. Assume that matrix equation (1.1) is consistent for Hermitian $R$-symmetric $X$, i.e., $A_{j} B_{j}^{*}=B_{j} A_{j}^{*}$ and $A_{j} A_{j}^{+} B_{j}=B_{j}$ for $j=1,2$, then,

$$
\begin{aligned}
\max _{A X=B, X^{*}=X, R X R=X} r(X) & =s+t-r\left(A_{1}\right)-r\left(A_{2}\right)+r\left(B_{1}\right)+r\left(B_{2}\right), \\
\min _{A X=B, X^{*}=X, R X R=X} r(X) & =2 r\left(B_{1}\right)+2 r\left(B_{2}\right)-r\left(A_{1} B_{1}^{*}\right)-r\left(A_{2} B_{2}^{*}\right), \\
\max _{A X=B, X^{*}=X, R X R=X} i_{ \pm}(X) & =s+t-r\left(A_{1}\right)-r\left(A_{2}\right)+i_{ \pm}\left(A_{1} B_{1}^{*}\right)+i_{ \pm}\left(A_{2} B_{2}^{*}\right), \\
\min _{A X=B, X^{*}=X, R X R=X} i_{ \pm}(X) & =r\left(B_{1}\right)+r\left(B_{2}\right)-i_{\mp}\left(A_{1} B_{1}^{*}\right)-i_{\mp}\left(A_{2} B_{2}^{*}\right) .
\end{aligned}
$$

To this end, we now investigate the extremal ranks and inertias of the Hermitian $R$-skew symmetric LS solutions to matrix equation (1.1). Define

$$
\mathcal{S}_{3}=\left\{X \mid\|A X-B\|=\min , X \in \mathbb{C}_{h}^{n} \text { and } R X R=-X\right\}
$$

Theorem 4.2. Suppose that the conditions are the same as in Theorem 4.1.

$$
\begin{aligned}
\text { Let } \quad T_{31}=\left[\begin{array}{cccc}
0 & A_{2} A_{2}^{*} A_{2} & 0 & -A_{2} \\
A_{1}^{*} B_{2} A_{2}^{*} & 0 & A_{1}^{*} A_{1} A_{1}^{*} & 0 \\
A_{2}^{*} A_{2} A_{2}^{*} & 0 & 0 & A_{2}^{*} A_{2} \\
0 & F_{A_{1}} B_{1}^{*} A_{2} & A_{1}^{*} & -\Omega_{1} T_{3} \Omega_{2}^{*}
\end{array}\right], \text { then, } \\
\max _{X \in \mathcal{S}_{3}} r(X)=2 \min \left\{s+s_{1}-2 r\left(A_{1}\right), t+r\left(T_{31}\right)-r\left(A_{1}\right)-3 r\left(A_{2}\right)\right\}, \\
\min _{X \in \mathcal{S}_{3}} r(X)=2\left[r\left(T_{31}\right)+s_{1}-s_{2}-r\left(A_{1}\right)-2 r\left(A_{2}\right)\right],
\end{aligned}
$$




$$
\begin{aligned}
& \max _{X \in \mathcal{S}_{3}} i_{ \pm}(X)=\min \left\{s+s_{1}-2 r\left(A_{1}\right), t+r\left(T_{31}\right)-r\left(A_{1}\right)-3 r\left(A_{2}\right)\right\}, \\
& \min _{X \in \mathcal{S}_{3}} i_{ \pm}(X)=r\left(T_{31}\right)+s_{1}-s_{2}-r\left(A_{1}\right)-2 r\left(A_{2}\right),
\end{aligned}
$$

where $s_{1}=r\left[\begin{array}{cc}A_{1}^{*} A_{1} A_{1}^{*} & A_{1}^{*} B_{2} \\ A_{1}^{*} & \Omega_{1} T_{3} \Omega_{2}^{*}\end{array}\right]$, and $s_{2}=r\left[\begin{array}{cc}A_{1}^{*} A_{1} A_{1}^{*} & A_{1}^{*} B_{2} A_{2}^{*} \\ A_{1} A_{1}^{*} & A_{1} \Omega_{1} T_{3} \Omega_{2}^{*} A_{2}^{*}\end{array}\right]$.

Proof. From (4.2), we know that

$$
r(X)=2 r\left(X_{3}\right) \text { and } i_{ \pm}(X)=r\left(X_{3}\right) .
$$

Thus it is enough to find the extremal ranks of $X_{3}$ as given in (4.7).

In fact, applying Lemma 2.7 to $X_{3}$, we get

$$
\begin{aligned}
& \max _{H_{3} \in \mathbb{C}^{s \times t}} r\left(X_{3}\right)=\min \left\{r\left[-L F_{A_{1}}\right], r\left[\begin{array}{c}
-L \\
F_{A_{2}}
\end{array}\right]\right\} \\
& \min _{H_{3} \in \mathbb{C}^{s \times t}} r\left(X_{3}\right)=r\left[-L F_{A_{1}}\right]+r\left[\begin{array}{c}
-L \\
F_{A_{2}}
\end{array}\right]-r(K) .
\end{aligned}
$$

where $K=\left[\begin{array}{cc}-L & F_{A_{1}} \\ F_{A_{2}} & 0\end{array}\right], L=\bar{X}_{3}-\Omega_{1} T_{3} \Omega_{2}^{*}$.

Now we simplify the above items by the elementary block matrix operations. Note that $A_{1}^{+} A_{1} \Omega_{1} T_{3} \Omega_{2}^{*}=\Omega_{1} T_{3} \Omega_{2}^{*}=\Omega_{1} T_{3} \Omega_{2}^{*} A_{2}^{+} A_{2}$, Lemma 2.2, (2.1) and (2.2) in Lemma 2.5 follow that

$$
r\left[-L F_{A_{1}}\right]=s-r\left(A_{1}\right)+r\left[\Omega_{1} T_{3} \Omega_{2}^{*}-A_{1}^{+} B_{2}\right]=s+s_{1}-2 r\left(A_{1}\right),
$$

and

$$
\begin{aligned}
r\left[\begin{array}{c}
-L \\
F_{A_{2}}
\end{array}\right]= & t-r\left(A_{2}\right)+r\left[A_{1}^{+} B_{2} A_{2}^{+} A_{2}+F_{A_{1}}\left(A_{2}^{+} B_{1}\right)^{*}-\Omega_{1} T_{3} \Omega_{2}^{*}\right] \\
= & {\left[\begin{array}{ccccc}
A_{2} & 0 & A_{2} A_{2}^{*} A_{2} & 0 & 0 \\
0 & A_{1}^{*} B_{2} A_{2}^{*} & 0 & A_{1}^{*} A_{1} A_{1}^{*} & 0 \\
I_{t} & 0 & 0 & 0 & I_{t} \\
0 & A_{2}^{*} A_{2} A_{2}^{*} & 0 & 0 & A_{2}^{*} A_{2} \\
0 & 0 & F_{A_{1}} B_{1}^{*} A_{2} & A_{1}^{*} & -\Omega_{1} T_{3} \Omega_{2}^{*}
\end{array}\right] } \\
& -r\left(A_{1}\right)-3 r\left(A_{2}\right) \\
= & t+r\left(T_{31}\right)-r\left(A_{1}\right)-3 r\left(A_{2}\right) .
\end{aligned}
$$

Moreover,

$$
\begin{aligned}
r(K) & =r\left[\begin{array}{ccc}
-L & I_{s} & 0 \\
I_{t} & 0 & A_{2}^{*} \\
0 & A_{1} & 0
\end{array}\right]-r\left(A_{1}\right)-r\left(A_{2}\right) \\
& =s+t+r\left(A_{1} \Omega_{1} T_{3} \Omega_{2}^{*} A_{2}^{*}-A_{1} A_{1}^{+} B_{2} A_{2}^{*}\right)-r\left(A_{1}\right)-r\left(A_{2}\right) \\
& =s+t+s_{2}-2 r\left(A_{1}\right)-r\left(A_{2}\right) .
\end{aligned}
$$

Therefore, substituting (4.20)-(4.22) into (4.18) and (4.19) and combining with (4.17) deduce that (4.13)-(4.16) hold. 
From Lemma 4.2, $A_{1} B_{1}^{*}=B_{2} A_{2}^{*}$ implies that $T_{3}=0$, in this case, the $r\left(T_{31}\right)$ in Theorem 4.2 can be written as

$$
r\left(T_{31}\right)=r\left[\begin{array}{ccc}
A_{1}^{*} A_{1} B_{1}^{*} & 0 & 0 \\
A_{2}^{*} A_{2} A_{2}^{*} & \left(A_{2}^{*} A_{2}\right)^{2} & 0 \\
0 & B_{1}^{*} A_{2} & A_{1}^{*}
\end{array}\right]+r\left(A_{2}\right) \doteq r\left(T_{31}^{\prime}\right)+r\left(A_{2}\right),
$$

and

$$
s_{1}=r\left(A_{1}\right)+r\left(A_{1}^{*} B_{2}\right) \text { and } s_{2}=r\left(A_{1}\right)+r\left(A_{1}^{*} A_{1} B_{1}^{*}\right) .
$$

Then we have the following result:

Corollary 5. Suppose that $A_{1} B_{1}^{*}=B_{2} A_{2}^{*}$ in Theorem 4.2 and $T_{31}^{\prime}$ as in (4.23), then,

$$
\begin{aligned}
\max _{X \in \mathcal{S}_{3}} r(X) & =2 \min \left\{s+r\left(A_{1}^{*} B_{2}\right)-r\left(A_{1}\right), t+r\left(T_{31}^{\prime}\right)-r\left(A_{1}\right)-2 r\left(A_{2}\right)\right\}, \\
\min _{X \in \mathcal{S}_{3}} r(X) & =2\left[r\left(T_{31}^{\prime}\right)+r\left(A_{1}^{*} B_{2}\right)-r\left(A_{1}^{*} A_{1} B_{1}^{*}\right)-r\left(A_{1}\right)-r\left(A_{2}\right)\right], \\
\max _{X \in \mathcal{S}_{3}} i_{ \pm}(X) & =\min \left\{s+r\left(A_{1}^{*} B_{2}\right)-r\left(A_{1}\right), t+r\left(T_{31}^{\prime}\right)-r\left(A_{1}\right)-2 r\left(A_{2}\right)\right\}, \\
\min _{X \in \mathcal{S}_{3}} i_{ \pm}(X) & =r\left(T_{31}^{\prime}\right)+r\left(A_{1}^{*} B_{2}\right)-r\left(A_{1}^{*} A_{1} B_{1}^{*}\right)-r\left(A_{1}\right)-r\left(A_{2}\right) .
\end{aligned}
$$

Since the consistency of matrix equation (1.1) under Hermitian $R$-skew symmetric constraint is coincided with that of matrix equations (4.5), if matrix equations (4.5) are consistent, that is, $A_{1} A_{1}^{+} B_{2}=B_{2}, A_{2} A_{2}^{+} B_{1}=B_{1}$ and $A_{1} B_{1}^{*}=B_{2} A_{2}^{*}$, then we have $r\left(A_{1}^{*} B_{2}\right)=r\left(B_{2}\right)$ and $r\left(A_{2}^{*} B_{1}\right)=r\left(B_{1}\right)$. Hence we can also obtain similar conclusion as in Corollary 2 .

Remark 4.1. Similar to the statements in remark 3.1, the extremal ranks and inertias of the Hermitian $R$-symmetric and $R$-skew symmetric LS solutions to matrix equations $A X=B, X C=D$ can also be derived by Theorems 4.1 and 4.2 , respectively.

\section{REFERENCES}

1. F. Uhlig, On the matrix equation $A X=B$ with applications to the generators of controllability matrix. Linear Algebra Appl., 85 (1987), 203-209.

2. M. Mesbahi, On the rank minimization problem and its control applications, Systems Control Lett., 33 (1998), 31-36.

3. C. Davis, Completing a matrix so as to minimize its rank, Oper. Theory: Adv. Appl., 29 (1988), 87-95.

4. S.K. Mitra, Fixed rank solutions of linear matrix equations. Sankhya, Ser. A., 35 (1972), 387-392.

5. G. Marsaglia, G.P.H. Styan, Equalities and inequalities for ranks of matrices, Linear Multilinear Algebra, 2 (1974) 269-292.

6. Y. Tian, The Solvability of two linear matrix equations, Linear Multilinear Algebra 48 (2000), 123-147.

7. M.S. Wei, Q.W. Wang, On rank-constrained Hermitian nonnegtive-definite least squares solutions to the matrix equation $A X A^{*}=B$, Int. J. Comput. Math., 84 (2007), 945-952. 
8. M. Mahajan, J. Sarma, On the complexity of matrix rank and rigidity, Lect. Notes Comput. Sci. Eng., 4649 (2007), 269-280.

9. Q.F. Xiao, X.Y. Hu, L. Zhang, The symmetric minimal rank solution of the matrix equation $A X=B$ and the optimal approximation, Electronic J. Linear Algebra, 18 (2009), 264-273.

10. Q. Zhang, Q.W. Wang, The $(P, Q)$-(skew)symmetric extremal rank solutions to a system of quaternion matrix equations, Appl. Math. Comput., 217 (2011) 9286-9296.

11. Y. Tian, Y. Liu, Extremal ranks of some symmetric matrix expressions with applications, SIAM J. Matrix Anal. Appl., 28 (2006), 890-905.

12. Y. Tian, Equalities and inequalities for inertias of Hermitian matrices with applications, Linear Algebra Appl., 433 (2010), 263-296.

13. Y. Tian. Maximization and minimization of the rank and inertia of the Hermitian matrix expression $A-B X-(B X)^{*}$ with applications, Linear Algebra Appl., 434 (2011), 2109-2139.

14. Y. Tian, On an equality and four inequalities for generalized inverses of Hermitian matrices, Electronic J. Linear Algebra, 23 (2012), 11-42.

15. Y. Tian, Solving optimization problems on ranks and inertias of some constrained nonlinear matrix functions via an algebraic linearization method, Nonlinear Anal.: Theory, Meth. Appl., 75:2 (2012), 717-734.

16. Y.H. Liu, Ranks of least squares solutions of the matrix equation $A X B=C$, Comput. Math. Appl., 55 (2008), 1270-1278.

17. F.X. Zhang, Y. Li, W.B. Guo, J.L. Zhao, Least squares solutions with special structure to the linear matrix equation $A X B=C$, Appl. Math. Comput., 217:24 (2011), 10049-10057.

18. F.X. Zhang, Y. Li, J.L. Zhao, Common Hermitian least squares solutions of matrix equations $A_{1} X A_{1}=B_{1}$ and $A_{2} X A_{2}=B_{2}$ and subject to inequality restrictions, Comput. Math. Appl. 62:6 (2011), 2424-2433.

19. Ying Li, Yan Gao, Wenbin Guo, A Hermitian least squares solution of the matrix equation $A X B=C$ subject to inequality restrictions, Comput. Math. Appl., 64:6 (2012), 1752-1760.

20. W.F. Trench, Hermitian, hermitian R-symmetric, and hermitian R-skew symmetric Procrustes problems, Linear Algebra Appl. 387 (2004), 83-98.

21. Y. Tian, Rank equalities related to generalized inverses of matrices and their applications, Master Thesis, Montreal, Quebec, Canada, 2000. http://arxiv.org/abs/math/0003224vl.

Lifang Dai received her master degree from Lanzhou University under the direction of Nonliear functional Analysis. Since 2009 she has been working at Tianshui Normal University of Gansu province.

School of Mathematics and Statistics, Tianshui Normal University, Tianshui Gansu 741001, P.R. of China.

e-mail:dailf2005@163.com

Maolin Liang received his master degree from Lanzhou University under the direction of Numerical Algebra and Applied Software. Since 2007 he has been working at Tianshui Normal University of Gansu province. His research interests is numerical linear algebra.

School of Mathematics and Statistics, Tianshui Normal University, Tianshui Gansu 741001, P.R. of China.

e-mail: liangm12005@163.com 